\title{
Roadblocks to PrEP: What Medical Records Reveal About Access to HIV Pre-exposure Prophylaxis
}

\author{
Avy A. Skolnik, $P h D^{1,2}$ (D), Barbara G. Bokhour, $P h D^{1,3}$, Allen L. Gifford, $M D^{4,5}$, \\ Brigid M. Wilson, $P h D^{6}$, and Puja Van Epps, $M D^{6,7}$
}

\begin{abstract}
'Center for Healthcare Organization and Implementation Research (CHOIR), ENRM Veterans Affairs Medical Center Bedford, Bedford, MA, USA; ${ }^{2}$ University Health Services, University of Massachusetts, Amherst, MA, USA; ${ }^{3}$ Department of Health Law, Policy, and Management, Boston University School of Public Health, Boston, MA, USA; ${ }^{4}$ Section of General Internal Medicine, Department of Medicine, Boston University School of Medicine, Boston, MA, USA; ${ }^{5}$ Center for Healthcare Organization and Implementation Research (CHOIR), VA Boston Healthcare System, Boston, MA, USA; ${ }^{6}$ Louis Stokes Cleveland Department of Veterans Affairs Medical Center (LSCVAMC), Cleveland, OH, USA; ${ }^{7}$ Department of Internal Medicine, Case Western School of Medicine, Cleveland, OH, USA.
\end{abstract}

BACKGROUND: Pre-exposure prophylaxis (PrEP) has been shown to be efficacious in preventing HIV; however, its uptake remains modest. Given that there are fewer cost barriers to receiving PrEP within VHA than via commercial insurance, VHA represents an ideal setting in which to study other barriers that may impact patients seeking PrEP.

OBJECTIVE: We sought to understand potential barriers to obtaining PrEP within the Veterans Health Administration (VHA) through examination of documentation in electronic medical records.

DESIGN: Retrospective structured chart review, including chart abstractions of notes, referrals, and communications; content analysis of charts from a subsample of patients receiving PrEP in VHA.

PARTICIPANTS: One hundred sixty-one patients prescribed PrEP at 90 sites varying in PrEP prescribing rates. APPROACH: We extracted descriptive information and conducted a qualitative analysis of all PrEP-relevant free-text notes including who initiated the PrEP conversation (patient vs. provider), time interval between request and prescription, reasons for denying PrEP, and patient responses to barriers.

KEY RESULTS: Patients initiated 94\% of PrEP conversations and $35 \%$ of patients experienced delays receiving PrEP ranging from six weeks to 16 months. Over $70 \%$ of cases evidenced barriers to access. Barriers included provider knowledge gaps about PrEP, provider knowledge gaps about VHA systems related to PrEP, confusion or disagreement over clinic purview for PrEP, and provider attitudes or stigma associated with patients seeking PrEP. CONCLUSIONS: Although PrEP is recommended for HIV prevention in high-risk persons, many PrEP-eligible individuals faced barriers to obtaining a prescription. Current practices place substantial responsibility on patients to request and advocate for this service, in contrast to many other preventive services. Understanding the prevalence and content of PrEP knowledge gaps and attitudinal

Presentations An earlier version of this manuscript was presented for a symposium at the VA HSR\&D National Conference in Washington DC in 2017.

Received May 7, 2019

Accepted October 2, 2019

Published online November 8, 2019 barriers can inform organizational interventions to increase PrEP access and decrease HIV transmission.

KEY WORDS: HIV/AIDS; prevention; pre-exposure prophylaxis; patientprovider communication; primary care.

J Gen Intern Med 35(3):832-8

DOI: $10.1007 / \mathrm{s} 11606-019-05475-9$

(c) Society of General Internal Medicine (This is a U.S. government work and not under copyright protection in the U.S.; foreign copyright protection may apply) 2019

$\mathrm{W}$

ith nearly 40,000 new HIV infections in the USA annually, the primary prevention of HIV transmission remains a national priority ${ }^{1}$. The use of once daily emtricitabine/tenofovir disoproxil fumarate (FTC-TDF) as pre-exposure prophylaxis (PrEP) has demonstrated tremendous efficacy for preventing HIV transmission. When used consistently, PrEP decreases HIV transmission $73-100 \%$ of the time in high-risk populations ${ }^{2-4}$. Yet despite demonstrated efficacy, uptake remains low ${ }^{5-8}$. The Centers for Disease Control (CDC) estimates that only a minority of the potential 1.2 million adults in the USA who might benefit from PrEP are accessing it .

Following the July 2012 FDA approval of PrEP for the prevention of HIV, the Veterans Health Administration's (VHA) Pharmacy Benefits Management Service released a review of PrEP in July of 2013 supporting its use within VHA for prevention of HIV. Yet despite VHA guidelines supporting PrEP ${ }^{10}$, findings from a previous study suggest that there is likely considerable variation in PrEP access and prescribing practices, both at the facility and provider level, with possible under-utilization of PrEP within some VHA facilities ${ }^{11,12}$. Questions remain regarding reasons for these low prescribing rates.

To our knowledge, this is the first study examining barriers to PrEP within a national healthcare system. Several nonVHA studies have attempted to understand barriers to access and prescriber practices by surveying patients or providers ${ }^{8}$, 13-16. Providers report fears about toxicities, medication nonadherence, cost, and potential for increased high-risk behavior 
contribute to reluctance to prescribe $e^{8,15-18}$. While surveys may be useful in understanding prescriber-reported practices and attitudes, they do not reveal actual practice. Electronic health records (EHR) may provide further insight into how providers prescribe PrEP. Such records may be useful in understanding providers' thinking and attitudes, along with their PrEP prescribing practices. Further, they may provide insight into patient characteristics and responses when barriers arise. We performed a qualitative content analysis of charts of veterans receiving PrEP in the VHA and sought to determine what documentation about HIV risk and PrEP in the EHR might reveal about barriers to PrEP access.

\section{METHODS}

We conducted a detailed chart review of a subsample of clinical notes of patients who had been prescribed PrEP across VHA. The EHR utilized by the VHA is a centralized system that contains health information, visit records, providerprovider communications, and patient-provider communications. We then conducted a conventional content analysis of these notes and communications to better understand PrEP access.

\section{Sample Selection}

We located records of patients prescribed PrEP based on an algorithm identifying HIV and Hep B-negative patients prescribed FTC-TDF for at least two consecutive months with no other concurrent anti-retrovirals ${ }^{12}$. At the time of sample selection, a total of 825 veterans at 105 VHA facilities were identified that had been prescribed PrEP since 2012, the year of Federal Drug Administration (FDA) approval of PrEP. Cases identified through the algorithm were confirmed via chart review. We did not determine a specific sample size initially for the content analysis, but we expected there to be variation across facilities and regions. Therefore, we sampled all patients from low prescribing facilities. Sites and patients from the remaining strata were then selected using a combination of stratified purposeful and purposeful random sampling ${ }^{19}$.

\section{Site Selection}

The team selected 90 sites for detailed chart review. We first classified sites as high, medium, or low prescribers of PrEP based on the ratio of HIV-positive patients to PrEP patients (Table 1). CDC estimates as many as 1.2 million people at risk for HIV could benefit from PrEP, but currently less than $6 \%$ are accessing $i^{20,21}$. We surmised that facilities with higher HIV-positive populations ought to have more patients that would also benefit from PrEP and used a conservative percentage lower than the current access figures (1\%) as the cut off for low-prescribing sites. All low prescribing sites (38) were selected for review, with the rationale being that low
Table 1 Low PrEP Prescribing Facilities (Facilities with 1 PrEP Case or with Ratio $\leq 1$ PrEP pt:100 HIV pts $)(N=38$ Facilities and 68 Patients)

\begin{tabular}{|c|c|c|c|c|c|}
\hline $\begin{array}{l}\text { Number of } \\
\text { HIV+ } \\
\text { Patients }\end{array}$ & $\begin{array}{l}\mathrm{SE} \\
\text { region }\end{array}$ & $\begin{array}{l}\mathrm{NE} \\
\text { region }\end{array}$ & $\begin{array}{l}\text { MW } \\
\text { region }\end{array}$ & $\begin{array}{l}\text { W/NW } \\
\text { region }\end{array}$ & $\begin{array}{l}\text { SW } \\
\text { region }\end{array}$ \\
\hline $11-99$ & 1 rural & 4 rural & $\begin{array}{l}5 \text { rural } \\
1 \text { urban }\end{array}$ & 2 rural & 2 rural \\
\hline 100-199 & $\begin{array}{l}1 \text { rural } \\
1 \text { urban }\end{array}$ & 2 rural & 1 urban & - & - \\
\hline $200-299$ & $\begin{array}{l}1 \text { rural } \\
4 \text { urban }\end{array}$ & & 1 urban & - & - \\
\hline $300-399$ & $\begin{array}{l}2 \text { urban } \\
1 \text { rural }\end{array}$ & - & - & - & - \\
\hline $400-499$ & 4 urban & 2 urban & - & - & - \\
\hline $500-700$ & 1 urban & 2 urban & - & - & - \\
\hline
\end{tabular}

prescribing sites were potentially more likely to exhibit barriers. We then selected sites from the remaining levels (mid and high PrEP-prescribing) which represented each major region of the USA, resulting in 26 sites per level.

\section{Patient Selection}

Among the 90 sites, 1-2 patients per site were randomly selected, with a few exceptions. All PrEP cases $(N=68)$ were reviewed in the low prescribing sites. Because the majority of PrEP patients identified within VHA were cisgender males, we oversampled transgender and female patients (Table 2). Rural sites represented $45 \%$ of the total PrEP prescribing sites for both the total PrEP cohort and the qualitative sample. However, because all low prescribing sites and all sites with 1 PrEP case were sampled, rural veterans comprised only $12 \%$ of total PrEP cohort, vs. $46 \%$ of the qualitative sample.

Table 2 Who is Getting PrEP?

\begin{tabular}{lll}
\hline \hline Patient descriptives & $\begin{array}{l}\text { Total cohort } \boldsymbol{N}= \\
\mathbf{8 2 5}\end{array}$ & $\begin{array}{l}\text { Qualitative } \boldsymbol{N}= \\
\mathbf{1 6 1}\end{array}$ \\
\hline White & $472(56 \%)$ & $96(60 \%)$ \\
Black & $167(20 \%)$ & $29(18 \%)$ \\
Latina/o & $123(15 \%)$ & $18(11 \%)$ \\
Native American or Asian & $32(4 \%)$ & $7(4 \%)$ \\
American & & \\
Race/ethnicity not & $42(5 \%)$ & $11(7 \%)$ \\
documented & & \\
Male & $801(97 \%)$ & $146(92 \%)$ \\
Female & $18(2 \%)$ & $9(5 \%)$ \\
Transgender & $7(1 \%)$ & $6(3 \%)$ \\
Mean age & 41.2 & 41.7 \\
Facilities in urban settings & $57(54 \%)$ & $49(54 \%)$ \\
HIV risk: MSM, MSMW & $698(85 \%)$ & $119(77 \%)$ \\
HIV risk: MSW & $64(8 \%)$ & $5(3 \%)$ \\
HIV risk: WSM, TSM & $25(3 \%)$ & $15(9 \%)$ \\
HIV risk: injection drug use & $13(2 \%)$ & $10(7 \%)$ \\
HIV risk factor not & $28(4 \%)$ & $11(7 \%)$ \\
documented & & \\
\hline
\end{tabular}

Transgender, includes trans men and trans women; MSM, men who have sex with men; WSM, women who have sex with men; TSM, Trans persons who have sex with men. Gender identity may not have been accurately captured in patient charts, so this figure may be an underestimate of actual numbers of transgender patients 


\section{Subsampling of Clinical Notes}

We located the notes within each person's EHR that corresponded to the date of the initial PrEP prescription. We then performed a retrospective search within the chart to locate the initial record of a PrEP inquiry made by the patient, or initial PrEP recommendation made by a provider. Once this initial conversation was identified, we worked further back in the chart to identify any lab orders or clinic visits, locating the earliest documentation of the patient's risk for contracting HIV. Within the time interval between initial risk factor and index PrEP fill, all notes within the EHR with the potential for PrEP relevant information were reviewed (see Table 3 ).

\section{Chart Abstraction and Qualitative Analysis}

We conducted chart abstractions of all PrEP-relevant clinical notes and communications. First, descriptive information was extracted, such as patient demographics, HIV risk factor(s), provider type, and clinic type. A case was labeled as having a "delay" in prescribing if the time interval between the initial PrEP request and index prescription was longer than 30 days, as chart reviews revealed this is ample time for a provider to order an HIV test, receive results, inform patient, and write a prescription. Cases longer than 30 days were excluded if the time interval included a patient no-show or cancellation, a medical contraindication, or if notes indicated that a patient was deliberating about PrEP.

Conventional content analysis of all PrEP-relevant free-text notes was conducted to identify emergent codes capturing other aspects of care related to PrEP and HIV prevention ${ }^{22}$. Notes were coded primarily using open coding (e.g., language used by providers to characterize patients). However, directed content analysis and a priori coding was used specifically to identify barriers (e.g., provider-level, system-level) ${ }^{22}$. Three authors reviewed charts and codes were organized with their corresponding quotations using Microsoft Excel. Due to the volume of charts coded, full charts were not double coded; however, segments of charts were reviewed by multiple authors. Next, a higher conceptual level of coding occurred in which codes were grouped into categories (e.g., codes pertaining to provider reasons for PrEP refusal). Full charts were also categorized by which (if any) barriers to access they exhibited. Coding was iterative resulting in a codebook after the first 12 patient records. The remaining charts were coded,

Table 3 Chart Abstraction Data Elements

\begin{tabular}{|c|c|}
\hline Clinics reviewed & $\begin{array}{l}\text { Primary care, infectious disease, } \\
\text { behavioral health, substance abuse } \\
\text { treatment }\end{array}$ \\
\hline $\begin{array}{l}\text { Types of records } \\
\text { reviewed } \\
\text { Key dates } \\
\text { Who initiated } \\
\text { Documentation of } \\
\text { HIV risk }\end{array}$ & $\begin{array}{l}\text { Encounter notes, phone notes, secure } \\
\text { messages, consults/referrals } \\
\text { Date of PrEP request, Date of PrEP Rx } \\
\text { Provider, patient } \\
\text { Patient disclosures, treatment for bacterial } \\
\text { STI, patient requests HIV lab, patient } \\
\text { prescribed PEP }\end{array}$ \\
\hline
\end{tabular}

themes emerged from categorical codes, and code refinement continued, with discrepancies in coding discussed and resolved in team meetings.

\section{RESULTS}

While $55 \%$ of patient charts reflected relatively straightforward PrEP access, we identified four categories of barriers to obtaining PrEP in $45 \%$ of the 161 unique patient charts reviewed. In addition to barriers, several patterns in the chart timelines were identified that reveal the impact of barriers and characterize some of the individuals who ultimately do obtain PrEP despite the obstacles.

\section{Site Characteristics}

Out of 90 sites selected, $45 \%$ were in rural locations, $42 \%$ were low prescribers, and 58\% were medium or high prescribers. Sites also varied by size of HIV patient population served, ranging from 11 patients to over 700 , with $53 \%$ of the charts sampled from facilities each serving fewer than 300 HIV-positive patients (see Table 1).

\section{Patient Characteristics}

The majority (97\%) of the PrEP cohort consisted of male veterans whose primary risk factor for HIV was documented as having male sexual partners. Over half $(56 \%)$ were identified as white. The qualitative sample of 161 patients had similar demographic characteristics to the total cohort (see Table 3).

\section{Findings}

We identified four primary barriers to PrEP prescribing: provider knowledge gaps about PrEP, provider knowledge gaps about PrEP-related VHA systems, provider attitudes about PrEP, and clinic purview barriers (see Table 4). We also identified patterns in the timelines of veterans receiving PrEP: evidence of patients initiating conversations about PrEP and delays in prescribing. Lastly, we identified persistence and PrEP knowledge as two qualities consistently characterizing patients who received PrEP.

\section{Barriers to PrEP Prescribing}

Barriers to PrEP were identified within EHR encounter notes, provider-provider communications, and provider-patient communications. We identified four themes associated with PrEP barriers: provider knowledge gaps about PrEP, provider knowledge gaps about PrEP-related VHA systems, provider attitudes, and clinic purview barriers (see Table 4). Barriers were detected in both urban and rural settings, and irrespective of background HIV prevalence. These barriers were detected within both primary care (PC) and infectious diseases (ID) clinics, sometimes even prior to a clinical encounter in interactions with nurses or with clerks. 
Table 4 Barriers to PrEP Access

\begin{tabular}{ll}
\hline \hline Theme & Example from EHR \\
\hline Provider PrEP knowledge gaps & $\begin{array}{l}\text { "Vet has been tested for HIV, was neg, I informed him this medication is not provided for preventive measures, } \\
\text { needs to protect himself by practicing safe sex and avoiding risky behaviors." } \\
\text { "Informed him that PrEP is effective only 50\% of the time, maybe less." } \\
\text { "(PrEP) is not part of standard practice at the VA at this time as a 'necessary medical care' and I would } \\
\text { recommend veteran go to the health department." } \\
\text { "I called pharm and they could not tell me whether Prep was available. I placed ID consult, but suspect it is } \\
\text { not available. I suspect consult to be d/c and informed patient of my expectations." } \\
\text { "I suggested a monogamous relationship." } \\
\text { "Multiple sexual partners, unable to maintain same sexual partner." } \\
\text { "I am not comfortable prescribing for this purpose." } \\
\text { "Patient called upset about receiving a letter from infectious disease clinic." } \\
\text { "(ID) Consult was d/c because pt is HIV neg. Please reschedule with primary care." }\end{array}$ \\
\hline
\end{tabular}

Provider knowledge gaps about PrEP were cases in which incorrect information about PrEP was communicated to patients or cases in which provider communications revealed knowledge gaps. Some encounter notes indicated that providers were unaware of PrEP, while others underestimated PrEP's effectiveness. In two cases, veterans attempted to make an appointment for the purpose of inquiring about PrEP and were told by clerks that such medication did not exist. Other knowledge gaps included incorrect dosing schedules provided to patients, or providers confusing PrEP with post-exposure prophylaxis (PEP). Providers required that patients obtain prescriptions from their partner's provider or provide documented evidence of their partners' HIV status, steps not recommended in either VHA or CDC guidelines and place a considerable burden on the patient.

Provider knowledge gaps about PrEP-related VHA systems also created barriers for veterans seeking PrEP. These were cases in which providers were misinformed about VHA policy or other systems related to PrEP. Some providers reported difficulty locating information about the availability of PrEP. Others assumed that VHA was not providing PrEP and advised patients to go elsewhere. Other providers assumed PrEP was not formulary or attempted to determine this by asking the pharmacy.

Provider attitudinal barriers were apparent in documentation of counseling of patients, particularly when paired with a refusal to prescribe PrEP. Some providers focused more on behavioral risk reduction strategies, suggesting limiting or even eliminating sexual activity and discouraging PrEP. In other cases, providers refused to prescribe PrEP to patients who appeared to be good candidates, without documenting a reason. Attitudinal barriers were also apparent in how some providers characterized patients seeking PrEP. One provider stated in a consult: "Veteran unwilling to change behaviors and would like to be evaluated for PrEP." This contrasts with more neutral encounter notes, for example: "patient reports multiple sexual partners, may be at increased risk for HIV." Some providers also used the term "admitted" in describing patient behaviors: "patient admits to homosexual behavior." An "admission" (as opposed to a "report" or "disclosure") implies culpability or that a behavior is problematic.
Clinic purview barriers were a systems issue identified in cases in which lack of agreement within facilities about the location of care for both PrEP initiation and PrEP maintenance led to delays, multiple visits, and patients being "bounced back and forth between primary care and HIV clinics." Most veterans initially inquired about PrEP in primary care; however, primary care providers often placed consults to ID clinics. In some cases, referral to ID clinics was upsetting or confusing to HIV-negative veterans. For some, visiting ID clinics or making appointments for PrEP posed privacy concerns.

\section{Patterns in Prescribing}

Onus on patients to request PrEP. At least $16 \%$ of the veterans who requested PrEP had documented historical evidence of elevated risk for contracting HIV, including being treated for a sexually transmitted infection, being prescribed PEP, requesting multiple HIV tests, or previously disclosing sharing needles or having an HIV-positive partner. Yet despite the prevalence of documented HIV risk, the majority (88\%) of patient charts had clear documentation that the patient initiated the PrEP request, while 10 charts $(6 \%)$ documented providerinitiated PrEP conversations. There were nine charts in which the PrEP initiator could not be identified. In most cases, providers noted that the patient was requesting it: "Homosexual male asking to be put on PrEP kit of Truvada." In other cases, this was recorded in the referral placed from primary care to ID clinics: "Pt requesting HIV prophylaxis for being homosexual and at high risk for HIV." Still other times, internal messages to providers from medical assistants, nursing staff, or clerks recorded the patient request: "Patient also stated interest in pre-exposure medications for HIV???? Please advise."

Patients face refusals and delays. In $35 \%$ of cases, barriers resulted in delays in obtaining PrEP prescriptions, with the interval between request and fill ranging from five weeks to 16 months. Reasons associated with delays were not always documented. In response to delays or refusals to prescribe PrEP, some patients made follow-up requests. It is not known what percentage of these requests went undocumented in the 
EHR but of the 142 veterans who made an initial PrEP inquiry, $23 \%$ of them had a second request documented in the EHR at a later medical visit or via the EHR electronic messaging system. Eleven patients (8\%) requested PrEP three or more times before obtaining a prescription.

\section{Site Variation}

While a full exploration of similarities and differences across low, medium, and high prescribing sites would require additional data not gathered for this study, a few patterns are worth noting. Providers at high prescribing sites tended to exhibit more thorough documentation of safer sex counseling and use less judgmental language in their documentation. They also tended to exhibit fewer knowledge gaps about VHA systems related to PrEP. It was interesting to note however that knowledge gaps about PrEP and attitudinal barriers were identified in all three types of sites. Cases of easeful PrEP access could also be located in low prescribing sites.

\section{Patients Exhibit Knowledge and Persistence}

Patients who obtained PrEP tended to enter the clinical encounter already educated about PrEP. Providers documented, for example, "Patient has been reading CDC guidelines," or "He brought in a journal article." Providers noted sources of patient PrEP awareness, including media, websites, friends, partners, non-VHA providers, and patients who themselves work in healthcare or public health.

Persistence, the ability and willingness to keep trying even after being turned away, also emerged as a theme characterizing patients in this study. Patients not only made multiple requests but they also made multiple clinic visits, requested appointments even when they were told PrEP was unavailable, utilized different strategies when requesting PrEP, and sought PrEP at VHA after being unable to obtain it elsewhere.

Both persistence and knowledge about PrEP were evident in the secure messages recorded in patients' EHR. After making a documented three PrEP inquiries spanning 13 months with no apparent follow-up from providers, one veteran wrote:

"Hi Dr., I am following up regarding the inquiry I made last year during my annual check-in about being prescribed Truvada, aka PrEP. What were you able to find out because I think I would be a good candidate for it. I still understand that it is another tool to prevent one from getting HIV and I should still use condoms just as women use birth control pills and men condoms to reduce pregnancies. Last time we spoke you said you would ask the HIV specialist there about it. Could you please let me know what was decided?"

Another patient record indicated she had requested PrEP and had not been informed for several weeks about whether it would be prescribed, despite receiving her negative HIV result:

"I received the test results. I'm aware that they were negative, and I would like to commence taking the medication by the name of Pre-Exposure Prophylaxis (PrEP). My partner was tested and was HIV positive, and that's why I decided to get tested. My partner and I have discussed taking the proper precautions. He, too, has made the right steps to begin the treatment that he needs. As you know my medical history, I would like if you can consider starting me on PrEP."

\section{DISCUSSION}

Although VHA was an early adopter of PrEP, many providers appear to remain unaware of the VHA approval of PrEP even years later. Overall, these findings suggest the vast majority of veterans who eventually obtain PrEP were able to do so because they (1) were already educated about PrEP, (2) were willing to disclose risk factors in inquiring about PrEP, and (3) were persistent advocates when such access was denied. This finding was corroborated by recent research in non-VHA settings ${ }^{13}$. While veterans in our sample did receive PrEP, many who were eligible for PrEP faced significant delays, placing them at continued risk for HIV. These individuals were eventually able to access PrEP only by surmounting systemslevel and/or provider-level barriers in knowledge and attitudes via persistent and articulate self-advocacy. This may be indicative of a disparity whereby only those with access to information about PrEP and enough self-assurance to advocate will get access.

This raises the question of what happens when patients lack the time, energy, money, education, mental health, ability, or self-assurance to be such an advocate. Previous studies have documented gender and racial disparities in PrEP access, noting that white men have disproportionately benefitted from PrEP in the USA, even though the highest rates of new infections are among communities of color ${ }^{23}$. VHA cohort demographics reflect the general population of individuals currently receiving PrEP in the USA, suggesting that the barriers identified in this study may be contributing to racial and gender disparities in access within $\mathrm{VHA}^{21}$.

Even strong self-advocates sometimes found themselves waiting over a year before obtaining PrEP. Such delays result not only in ongoing HIV exposure risk, but may also sour the patient experience of their healthcare. This burden on patients also deviates from other forms of preventative care (e.g., immunizations, cancer screenings) in which patients are not expected to request. Placing the majority of the onus on the patient to request this service may be contributing to the modest uptake of PrEP in VHA and other settings.

Notably, provider attitudes towards individuals seeking PrEP were visible in the EHR documentation. Most apparent was a reluctance to prescribe when patients disclosed multiple sexual partners, even though this is an indicator for PrEP ${ }^{1}$. This barrier did not emerge in most previous studies about provider PrEP attitudes ${ }^{24}$. Negative attitudes towards samesex sexual behavior were also apparent in this study, but not reflected in previous PrEP studies ${ }^{8,14,16,24}$. Previous studies 
on provider self-reported attitudes about PrEP note a reluctance to prescribe due to fears about toxicity and medication non-adherence, but these concerns did not emerge in this study. Interestingly, a recent VHA study noted providers' moral judgments of HIV-positive patients they perceived as non-adherent to HIV medications. Such patients were sometimes characterized as "flakey" or even "socio-paths" by some providers ${ }^{25}$. Outside of the HIV literature, parallels can also be found in the moral discourse research in the prescribing of emergency contraception (EC $)^{26,27}$. One study, which focused on patient and provider attitudes towards pre-emptive EC prescribing, found that many providers expressed reluctance to proactively prescribe $\mathrm{EC}$, believing it may increase risky sexual behavior and STI transmission ${ }^{26}$. Patients not in stable, monogamous relationships were deemed "inappropriate candidates," a moral judgment echoed in our study.

This study had certain limitations. Because we utilized the algorithm to identify cases, this analysis was limited to patients who were eventually able to access PrEP. To fully understand the scope of the barriers to PrEP, reviewing records from patients who sought but were never able to obtain PrEP, as well as records in which providers recommended PrEP but patients declined it, would be ideal. As a result, this study may underestimate barriers to PrEP access. Additionally, while examining EHR allows the gathering of qualitative data without relying upon participant recall or self-report, EHR documentation may not fully reflect the content of an actual clinical encounter. Patient volume, clinic demands, and other variables could have interfered with thoroughness or accuracy of documentation. Verbatim provider-patient communications are only available via messaging, which provided a valuable record of the patient voice in this study. Lastly, chart reviews do not allow for follow-up or clarifying questions to be asked of either providers or patients. Future studies utilizing semistructured interviews of both groups would provide valuable additional insights, particularly into the veteran experience of seeking PrEP.

\section{Implications and Recommendations}

Increase Capacity for PrEP Access in Primary Care. Educational interventions which increase primary care providers' knowledge about PrEP and comfort counseling about sexual practices are likely to increase PrEP access, as studies have found that primary care settings have a greater capacity for and access to patients at risk for HIV ${ }^{15,17}$. Studies have also found an association of high levels of PrEP awareness with increased willingness to prescribe $\operatorname{PrEP}^{16,28}$, 29. Patients in this study gravitated towards primary care for PrEP citing privacy, appointment availability, and clinic proximity. In numerous facilities, provider confusion about location of care for PrEP delayed care. Findings in this study corroborated the "purview paradox" which has been described in previous studies, whereby HIV providers believe PrEP should be accessed in primary care, while primary care providers express concerns with initiating about maintaining patients on ARVs ${ }^{15}, 17,24$. National VHA guidelines lifting restrictions on primary care prescribing of PrEP will help decrease confusion about location of care. Education of nonclinical primary care staff about the availability of this service will help decrease the chance that veterans will be turned away from care or experience confidentiality concerns when inquiring about PrEP.

Implement Educational Initiatives That Address Bias. Attitudinal barriers were apparent in both ID and PC settings. Education about harm reduction approaches and ways to non-judgmentally counsel patients about risk factors may be helpful in addressing these barriers. Additionally, educational interventions for both primary care and ID providers that address implicit bias may and its impact on the clinical encounter may improve PrEP access and the experience of care, especially for veterans of color and LGBT veterans seeking $\operatorname{PrEP}^{30,} 31$.

Improve identification of PrEP candidates. A national VHAwide risk identification tool has recently been developed which can be implemented as a clinical reminder to evaluate for PrEP. However, it is seldom utilized, in part because providers may not be aware of it. Increasing the visibility and awareness of the tool among providers may help decrease the onus on the patient to raise the issue of HIV risk by prompting the clinician to inquire about risk and provide prevention options.

\section{CONCLUSION}

Chart reviews of encounter narratives, consults, and communications constructed an informative story about the patient experience and process of care associated with HIV prevention and PrEP. Patterns that emerged across multiple patients' records, as well as within the timeline of a single patient chart, provided a valuable picture of PrEP access within VHA. Clinical and systemic knowledge gaps, stigma and attitudinal barriers, and the veracity of patient advocacy in the desire to protect oneself from HIV may be indicative of PrEP-relevant themes outside VHA as well. These findings can inform targeted approaches that are needed to improve PrEP access to those at risk for HIV infection.

Corresponding Author: Avy A. Skolnik, PhD; University Health Services University of Massachusetts, Amherst, MA, USA (e-mail: askolnik@umass.edu).

Funding Information Dr. Van Epps is supported by the Geriatric Research Education and Clinical Center (GRECC). Additionally, Dr. Skolnik was funded by a VHA post-doctoral research fellowship.

\section{Compliance with Ethical Standards:}

Conflict of Interest: No conflict of interest, financial or other, exists for any of the authors of this manuscript. 


\section{REFERENCES}

1. Pre-Exposure Prophylaxis (PrEP) | HIV Risk and Prevention | HIV/AIDS | CDC. https://www.cdc.gov/hiv/risk/prep/index.html. Published August 23, 2018. Accessed September 30, 2018.

2. Baeten JM, Donnell D, Ndase P, et al. Antiretroviral Prophylaxis for HIV1 Prevention among Heterosexual Men and Women. N Engl J Med 2012;367(5):399-410. https://doi.org/10.1056/NEJMoa1108524.

3. Grant RM, Lama JR, Anderson PL, et al. Preexposure Chemoprophylaxis for HIV Prevention in Men Who Have Sex with Men. N Engl J Med. 2010;363(27):2587-2599. https://doi.org/10.1056/NEJMoa1011205.

4. Fonner VA, Dalglish SL, Kennedy CE, et al. Effectiveness and safety of oral HIV preexposure prophylaxis for all populations. AIDS. 2016;30(12):1973-1983. https://doi.org/10.1097/QAD. 0000000000001145.

5. Kirby T, Thornber-Dunwell M. Uptake of PrEP for HIV slow among MSM. Lancet. 2014;383(9915):399-400. https://doi.org/10.1016/ S0140-6736(14)60137-9.

6. Krakower DS, Mimiaga MJ, Rosenberger JG, et al. Limited Awareness and Low Immediate Uptake of Pre-Exposure Prophylaxis among Men Who Have Sex with Men Using an Internet Social Networking Site. PLoS ONE. 2012;7(3):e33119. https://doi.org/10.1371/journal.pone. 0033119.

7. Grant RM, Anderson PL, McMahan V, et al. Uptake of pre-exposure prophylaxis, sexual practices, and HIV incidence in men and transgender women who have sex with men: a cohort study. Lancet Infect Dis. 2014;14(9):820-829. https://doi.org/10.1016/S1473-3099(14)70847-3.

8. Tellalian D, Maznavi K, Bredeek UF, Hardy WD. Pre-Exposure Prophylaxis (PrEP) for HIV Infection: Results of a Survey of HIV Healthcare Providers Evaluating Their Knowledge, Attitudes, and Prescribing Practices. AIDS Patient Care STDs. 2013;27(10):553-559. https://doi.org/10.1089/apc.2013.0173.

9. Vital Signs: Estimated Percentages and Numbers of Adults with Indications for Preexposure Prophylaxis to Prevent HIV Acquisition - United States, 2015. https://www.cdc.gov/mmwr/preview/mmwrhtml/ mm6446a4.htm. Accessed September 30, 2018.

10. US Public Health Service. Preexposure prophylaxis for the prevention of HIV infection in the United States-2014: a clinical practice guideline. Washington, DC: US Public Health Service; 2014.

11. Maier MM, Gylys-Colwell I, Lowy E, et al. Health Care Facility Characteristics are Associated with Variation in Human Immunodeficiency Virus Pre-exposure Prophylaxis Initiation in Veteran's Health Administration. AIDS Behav. 2018. https://doi.org/10.1007/s10461-0182360-6.

12. Garner W, Wilson BM, Beste L, Maier M, Ohl ME, Van Epps P. Gaps in Preexposure Prophylaxis Uptake for HIV Prevention in the Veterans Health Administration. Am J Public Health. 2018;108(S4):S305-S310. https://doi.org/10.2105/AJPH.2018.304788.

13. Sun CJ, Anderson KM, Bangsberg D, et al. Access to HIV Pre-exposure Prophylaxis in Practice Settings: a Qualitative Study of Sexual and Gender Minority Adults' Perspectives. J Gen Intern Med. 2019;34(4):535-543. https://doi.org/10.1007/s11606-019-04850-w.

14. Adams LM, Balderson BH. HIV Providers' Likelihood to Prescribe Preexposure Prophylaxis (PrEP) for HIV Prevention Differs By Patient Type: A Short Report. AIDS Care. 2016;28(9):1154-1158. https://doi.org/10. 1080/09540121.2016.1153595.

15. Krakower D, Ware N, Mitty JA, Maloney K, Mayer KH. HIV Providers' Perceived Barriers and Facilitators to Implementing Pre-Exposure Prophylaxis in Care Settings: A Qualitative Study. AIDS Behav. 2014;18(9):1712-1721. https://doi.org/10.1007/s10461-014-0839-3.

16. Blumenthal J, Jain S, Krakower D, et al. Knowledge is Power! Increased Provider Knowledge Scores regarding Pre-exposure Prophylaxis (PrEP) are Associated with Higher Rates of PrEP Prescription and Future Intent to Prescribe PrEP. AIDS Behav. 2015;19(5):802-810. https://doi.org/10. 1007/s10461-015-0996-Z.
17. Silapaswan A, Krakower D, Mayer KH. Pre-Exposure Prophylaxis: A Narrative Review of Provider Behavior and Interventions to Increase PrEP Implementation in Primary Care. J Gen Intern Med. 2017;32(2): 192-198. https://doi.org/10.1007/s11606-016-3899-4.

18. Puro V, Palummieri A, De Carli G, Piselli P, Ippolito G. Attitude towards antiretroviral pre-exposure prophylaxis (PrEP) prescription among HIV specialists. BMC Infect Dis. 2013;13(1):217. https://doi. org/10.1186/1471-2334-13-217.

19. Palinkas LA, Horwitz SM, Green CA, Wisdom JP, Duan N, Hoagwood K. Purposeful sampling for qualitative data collection and analysis in mixed method implementation research. Admin Pol Ment Health. 2015;42(5):533-544. https://doi.org/10.1007/s10488-013-0528-y.

20. Smith DK, Van Handel M, Grey J. Estimates of adults with indications for HIV pre-exposure prophylaxis by jurisdiction, transmission risk group, and race/ethnicity, United States, 2015. Ann Epidemiol. 2018;28(12):850-857.e9. https://doi.org/10.1016/j.annepidem.2018. 05.003.

21. Siegler AJ, Mouhanna F, Giler RM, et al. The prevalence of preexposure prophylaxis use and the pre-exposure prophylaxis-to-need ratio in the fourth quarter of 2017, United States. Ann Epidemiol. 2018;28(12):841-849. https://doi.org/10.1016/j.annepidem.2018.06. 005.

22. Hsieh H-F, Shannon SE. Three Approaches to Qualitative Content Analysis. Qual Health Res. 2005;15(9):1277-1288. https://doi.org/10. $1177 / 1049732305276687$.

23. Eaton LA, Driffin DD, Bauermeister J, Smith H, Conway-Washington C. Minimal Awareness and Stalled Uptake of Pre-Exposure Prophylaxis (PrEP) Among at Risk, HIV-Negative, Black Men Who Have Sex with Men. AIDS Patient Care STDs. 2015;29(8):423-430. https://doi.org/10.1089/ apc.2014.0303.

24. Turner L, Roepke A, Wardell E, Teitelman AM. Do You PrEP? A Review of Primary Care Provider Knowledge of PrEP and Attitudes on Prescribing PrEP. J Assoc Nurses AIDS Care. 2018;29(1):83-92. https://doi.org/10. 1016/j.jana.2017.11.002.

25. Fix GM, Hyde JK, Bolton RE, et al. The moral discourse of HIV providers within their organizational context: An ethnographic case study. Patient Educ Couns. 2018. https://doi.org/10.1016/j.pec.2018. 08.018.

26. Karasz A. The Visit Before the Morning After: Barriers to Preprescribing Emergency Contraception. Ann Fam Med. 2004;2(4):345-350. https:// doi.org/10.1370/afm.105.

27. Wallace JL, Wu J, Weinstein J, Gorenflo DW, Fetters MD. Emergency contraception: knowledge and attitudes of family medicine providers. Fam Med. 2004;36(6):417-422.

28. Mimiaga MJ, White JM, Krakower DS, Biello KB, Mayer KH. Suboptimal awareness and comprehension of published pre-exposure prophylaxis efficacy results among physicians in Massachusetts. AIDS Care. 2014;26(6):684-693. https://doi.org/10.1080/09540121.2013. 845289.

29. Wimberly YH, Hogben M, Moore-Ruffin J, Moore SE, Fry-Johnson Y. Sexual history-taking among primary care physicians. J Natl Med Assoc. 2006;98(12):1924-1929.

30. Calabrese SK, Earnshaw VA, Underhill K, Hansen NB, Dovidio JF. The Impact of Patient Race on Clinical Decisions Related to Prescribing HIV Pre-Exposure Prophylaxis (PrEP): Assumptions About Sexual Risk Compensation and Implications for Access. AIDS Behav. 2014;18(2):226-240. https://doi.org/10.1007/s10461-013-0675-x.

31. Sabin JA, Riskind RG, Nosek BA. Health Care Providers' Implicit and Explicit Attitudes Toward Lesbian Women and Gay Men. Am J Public Health. 2015;105(9):1831-1841. https://doi.org/10.2105/AJPH.2015. 302631 .

Publisher's Note Springer Nature remains neutral with regard to jurisdictional claims in published maps and institutional affiliations. 